\title{
Robotic Vehicles to Simulate RFID-based Vehicular Ad Hoc Networks (Poster Abstract)
}

\author{
J. Munilla, A. Ortiz, A. Peinado \\ University of Malaga \\ E.T.S.Ingenieria de Telecomunicacion \\ Campus de Teatinos, Malaga, Spain \\ Ph. +34 952131305
}

munilla@ic.uma.es, aortiz@ic.uma.es, apeinado@ic.uma.es

\begin{abstract}
The study of vehicular ad hoc networks (VANETs) is usually performed by means of software simulations. However, in order to evaluate the integration of Radio Frequency Identification (RFID) technology into VANETs functionality, we have developed a scale test bench. It is composed of several vehicles provided with an RFID reader to detect the tags on the road, and the wireless native capability of the VANETs to communicate with other vehicles. The main objective of this RFID-VANET simulator is to show the interoperation of these technologies as a previous step to real implementations in vehicles. For this reason, we have incorporated specific circuitry to the small robots, and programmed them to reproduce real scenarios.
\end{abstract}

\section{Categories and Subject Descriptors}

K.4.1 [Computers and Society]: Public Policy Issues - human safety

\section{General Terms}

Performance, Design, Experimentation, Verification.

\section{Keywords}

VANETs, RFID, Traffic safety.

\section{INTRODUCTION}

Vehicular ad hoc networks (VANETs) are becoming more popular as a way to increase the traffic safety and comfort [3]. These networks allow connectivity among mobile hosts while the interaction with a fixed infrastructure is not an essential requirement. This way, vehicles in a VANET can share information to each other in a short range by using the $802.11 \mathrm{p}$ wireless technology.

Permission to make digital or hard copies of all or part of this work for personal or classroom use is granted without fee provided that copies are not made or distributed for profit or commercial advantage and that copies bear this notice and the full citation on the first page. To copy otherwise, or republish, to post on servers or to redistribute to lists, requires prior specific permission and/or a fee.

SIMUTools 2010 March 15-19, Torremolinos, Malaga, Spain.

Copyright 2010 ICST, ISBN 78-963-9799-87-5.
There are two different types of information that can be used to improve the traffic safety. The first corresponds to information coming from other vehicles regarding traffic congestion status or accident alerting. This information is usually transmitted in broadcast mode and smart broadcast techniques have to be used in order not to overload the network by flooding. The second corresponds to environmental information coming from traffic signs, speed limits, motorway tolls or semaphores [1].

The use of RFID technology to collect environmental data requires reading tags deployed on the road with the reader located on the vehicle. The RFID infrastructure [2] consists of a series of RFID tags located at any signalling point. So, the tags have to contain information regarding the point in which they are located (i.e. a speed limit, a dangerous bend, etc.).

The study of VANETs is usually performed by means of software simulations. However, in order to evaluate the integration of RFID functionality into VANETs, we have developed a scale test bench composed of several vehicles provided with an RFID reader to detect the tags on the road and the wireless native capability of the VANETs to communicate with other vehicles.

\section{TEST-BENCH DESCRIPTION}

The main objective of this RFID-VANET simulator is to show the interoperation of these technologies as a previous step to real implementations in vehicles. For this reason, vehicles with autonomous driving capability are employed in the simulator, in order to reproduce real driving scenarios.

The simulator is composed of a test driving circuit, several vehicles, and a set of driving tests representing potential risks for traffic safety.

\subsection{Test driving circuit}

In order to test the behaviour of the traffic safety applications based on RFID and VANET, we have designed a road circuit composed of roundabouts, crossroads, speed limitation signals and one-way roads. The road circuit has been printed on 150 x 90 centimetres paper size.

The road circuit also incorporates RFID tags at significant locations, such as the approaching to crossroads or roundabouts, speed limitation signals or one-way roads. These tags are detected by the vehicles when they are passing over them. In this way, a 
wrong way warning can be sent to other vehicles or a collaborative algorithm can be performed between the vehicles accessing to crossroads or roundabouts. In this way, the interoperation between RFID and VANET technologies can be tested.

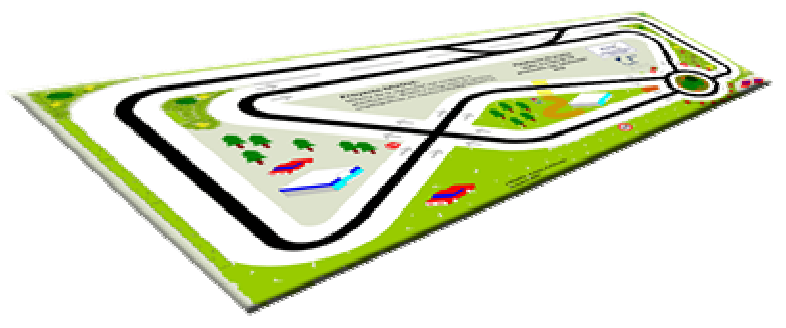

Figure 1. Test driving circuit

Additionally, some RFID tags are located on the road circuit to help to the autonomous driving process. These tags do not belong to the RFID-VANET protocol to be simulated, but to the own simulator to allow the robotic vehicles to drive on the road circuit.

\subsection{Robotic Vehicles}

The vehicles are little size robots with the ability to track a black line. This functionality is governed by a main PIC microcontroller and a secondary microcontroller dedicated to control the wheels movements.

The vehicles have been constructed using the educational Moway mini-robot as the starting point. An additional PCB with an RFID reader and a Zigbee transceiver has been designed, developed and incorporated to every robot. Finally, the RFID antenna has been integrated in the own robotic vehicle.

The robotic vehicles have been programmed to acquire information from the road by means of the RFID reader and information from others vehicles through the VANET protocol. This information is processed on board producing a variation or interruption of the movement and/or the generation of warning alerts to be sent to the others vehicles.

In a more general way, the vehicles can be programmed with the desired RFID-VANET protocol. In this way, the advantages of these applications can be shown to the open public.
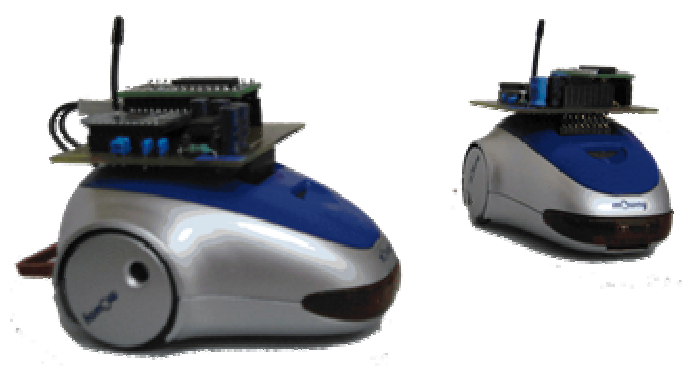

Figure 2. Robotic vehicles

\subsection{Driving tests}

Several tests have been performed in order to reproduce real scenarios with potential risk for traffic safety. These situations are crossroads, limitation of speed, wrong way and roundabouts.

The two roads entering the crossroad are signalled by RFID tags, but only one has preference. When the vehicle driving the nonpreference road reads the corresponding tag, it stops and listens the radio channel looking for another vehicle entering the crossroad. If no information is received, the vehicle enters the crossroad.

On the other hand, when vehicle driving the preference road reads the tag, it broadcasts a warning, and enters the crossroad.

In a similar way, vehicles can read RFID tag when they approximate the roundabout. At this state, a protocol over the VANET is established between the vehicles to avoid collisions and manage the traffic inside the roundabout.

Limitation of speed is also communicated to the vehicles through an RFID tag. When it drives over the signalling point, the automated vehicle reduces the speed. In a real situation, the system on board only informs to the driver. The speed reduction depends on the driver, not on the machine.

Another interesting scenario appears when a vehicle drives in the opposite direction. In this case, the vehicle can detect this situation reading a couple of tags. If the wrong way is detected, then the system on board broadcast a warning over the VANET. In this test bench, the vehicle automatically corrects the direction.

Finally, it is important to note that the robotic vehicles can be easily programmed to performed additional tests, using new paper road circuits.

\section{ACKNOWLEDGMENTS}

This work has been partly supported by the Spanish Ministry of Science and Innovation and the European FEDER funds under project TIN 2008-02236/TSI, "MUOVE".

\section{REFERENCES}

[1] Chiasserini, C., Fasolo, E., Furiato, R., Gaeta, R., Garetto, M., Gribaudo, M., Sereno, M., Zanella, A., 2005. Smart Broadcast of Warnning Messages in vehicular ad hoc applications. In Proceedings of Workshop Progetto NEWCOM (NoE) (Turin, Italy, November, 2005)

[2] Finkenzeller, K., 2002, RFID Handbook, second edition, Wiley

[3] Yousefi, S., Mousavi, M., Fathy, M., 2006. Vehicular ad hoc networks (VANETs): challenges and perspectives. In Proceedings of $6^{\text {th }}$ International Conference on ITS Telecommunications (Chegdu, China, June, 21-23, 2006) 761-766 DOI 10.1109/ITST.2006.289012 